\title{
Single-Particle Self-Excited Oscillator
}

\author{
B. D’Urso, ${ }^{*}$ R. Van Handel, ${ }^{\dagger}$ B. Odom, D. Hanneke, and G. Gabrielse \\ Department of Physics, Harvard University, Cambridge, Massachusetts 02138, USA
}

(Received 10 August 2004; published 23 March 2005)

\begin{abstract}
Electronic feedback is used to self-excite the axial oscillation of a single electron in a Penning trap. Large, stable, easily detected oscillations arise even in an anharmonic potential. Amplitudes are controlled by adjusting the feedback gain, and frequencies can be made nearly independent of amplitude fluctuations. Quantum jump spectroscopy of a perpendicular cyclotron motion reveals the absolute temperature and amplitude of the self-excited oscillation. The possibility to quickly measure parts per billion frequency shifts could open the way to improved measurements of $e^{-}, e^{+}, p$, and $\bar{p}$ magnetic moments.
\end{abstract}

DOI: $10.1103 /$ PhysRevLett.94.113002

PACS numbers: $32.80 . P j, 12.20 . F v, 42.50 . L c$

The harmonic motion of an oscillator can be excited and sustained with a driving force derived from its own oscillation. A wide range of macroscopic oscillators are operated as self-excited oscillators (SEOs), from the electromechanical clock [1] and its ubiquitous quartz successors, to the nanomechanical cantilevers used in atomic force microscopes [2] and sensitive electrometers [3]. A microscopic SEO is more difficult to realize because such small signals and driving forces are involved. The possibility of realizing a one-ion SEO in a Paul trap was once discussed [4], and self-driven feedback cooling of a oneelectron oscillator has been realized [5].

In this Letter we demonstrate a microscopic, oneparticle SEO for the first time. The axial motion of a single electron suspended in a Penning trap is driven by an electric field derived from the current that its motion induces in an electrical circuit. The principal challenge is in stabilizing the electron's oscillation amplitude, an amplitude measured here using quantum jump spectroscopy of a perpendicular cyclotron motion. The frequency stability and the signal-to-noise ratio allow detection of a five parts in $10^{10}$ frequency shift in a few seconds - a sensitivity that allows the detection of a one-quantum change in the electron cyclotron energy and an electron spin flip. Likely applications are improved measurements of the electron, positron, proton, and antiproton magnetic moments.

The oscillation which is self-excited is that of a single electron (charge $-e$ and mass $m$ ) along the central axis $(\hat{\mathbf{z}})$ of a cylindrical Penning trap [6] (Fig. 1) maintained at either 0.1 or $1.6 \mathrm{~K}$. A ring electrode at potential $-V_{0}$ with respect to grounded end caps generates a potential on the $z$ axis,

$$
\Phi(z)=\frac{V_{0}}{2}\left[C_{2} \frac{z^{2}}{d^{2}}+C_{4} \frac{z^{4}}{d^{4}}+C_{6} \frac{z^{6}}{d^{6}}+\cdots\right]
$$

where $d=0.35 \mathrm{~cm}$ indicates the trap size. The $C_{k}$ are determined by trap geometry and by the potential $V_{c}$ applied to small compensation electrodes (Fig. 1) to adjust
$C_{4}$ and $C_{6}$. An "orthogonalized" trap geometry [6] makes $C_{2}$ essentially independent of $V_{c}$.

A drive force $F_{d}(t)$ and a damping force $-m \gamma_{z} \dot{z}$ yield

$$
\ddot{z}+\gamma_{z} \dot{z}+\left[\omega_{z}(A)\right]^{2} z=F_{d}(t) / m .
$$

The angular oscillation frequency $\omega_{z}(A)$ is

$$
\frac{\omega_{z}(A)}{\omega_{z}} \approx 1+\frac{3 C_{4}}{4 C_{2}}\left(\frac{A}{d}\right)^{2}+\frac{15 C_{6}}{16 C_{2}}\left(\frac{A}{d}\right)^{4},
$$

for small $\left(C_{4} / C_{2}\right)^{2}$. It depends weakly upon the oscillation amplitude $A$ [7], and $\omega_{z}=\sqrt{e V_{0} C_{2} /\left(m d^{2}\right)}$ pertains for small amplitudes.

The motion induces a voltage proportional to $\dot{z}$ across a $Q \approx 600$ tuned circuit ( $R$ in Fig. 1). Energy dissipated in $R$ damps the motion. The signal is amplified with a high electron mobility transistor (Fujitsu FHX13LG) anchored to the cryogenic environment and operated at a very low current to minimize trap heating. For the two realizations of the SEO that we will describe, typically $420 \mu \mathrm{W}$ is used to detect the comparator SEO operated at $1.6 \mathrm{~K}$, and only

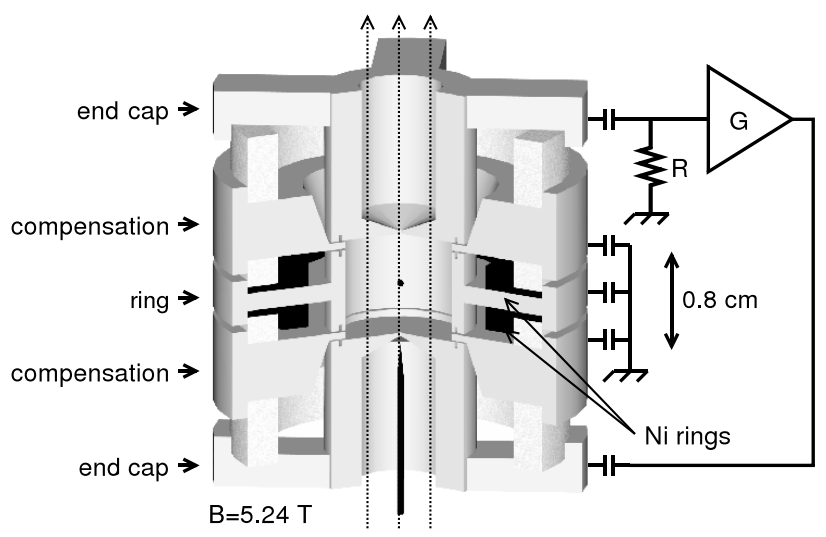

FIG. 1. The vertical oscillation of a trapped electron, shown within a cutaway of a cylindrical Penning trap, induces a voltage across resistor $R$ that is amplified and fed back to drive the oscillation. Unavoidable trap capacitance in parallel to $R$ is tuned out at $\omega_{z}$ with a parallel inductor. 
$12 \mu \mathrm{W}$ for the DSP (digital signal processor) SEO at $0.1 \mathrm{~K}$. Some amplified signal is phase shifted and fed back to the opposite end cap to drive the SEO. The rest is Fourier transformed to determine the SEO's amplitude and oscillation frequency.

The feedback produces a force $F_{d}(t)=G m \gamma_{z} \dot{z}$. Feedback cooling of the electron motion takes place if $G<1$ [5]. Self-excitation occurs, in principle, when the feedback cancels the damping, for unit feedback gain $G=1$. However, any noise will cause amplitude diffusion and energy growth. Also, if $G$ differs even slightly from unity, $A$ will either decrease or increase exponentially.

A stable and useful SEO thus requires a fixed oscillation amplitude $A_{o}$, arranged using an amplitude-dependent gain $G(A)$ that decreases with increasing $A$ near $G\left(A_{o}\right)=1$. This gain in Eq. (2) yields

$$
\dot{A}=-\frac{1}{2} \gamma_{z} A[1-G(A)]
$$

for the time evolution of the amplitude [8]. In practice, the gain-control system may average the signal for a time $\tau$ before determining $A$. Equation (4) is valid if $\tau \gg 1 / \omega_{z}$, and $1 / \tau$ is much larger than the resulting self-excited oscillator linewidth.

We demonstrate two methods of stabilizing the amplitude of a SEO-passing the feedback drive through a comparator [Fig. 2(a)] and employing a fast DSP [Fig. 2(b)]. The first was realized at $\omega_{z} /(2 \pi)=64 \mathrm{MHz}$ and $1.6 \mathrm{~K}$ and the second at $\omega_{z} /(2 \pi)=200 \mathrm{MHz}$ and $0.1 \mathrm{~K}$ for reasons not related to this demonstration. The comparator is simpler, but the DSP is the more flexible option that can be made much more immune to noise. For both demonstrations the technical noise added by the feedback amplifier is so small [5] that we neglect it in our analysis. We were unable with the electrodes of our trap to realize a third method-applying the signal induced on one electrode to a second electrode (not the image of the first under $z \rightarrow-z$ ) to make the effective feedback gain decrease with oscillation amplitude.
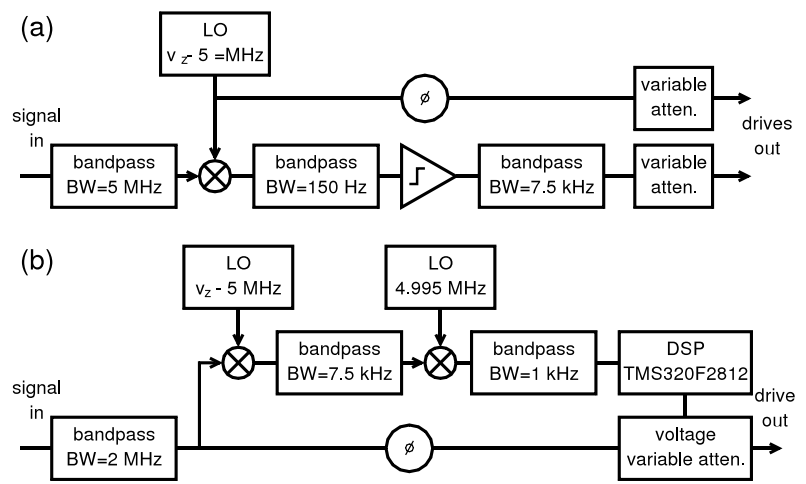

FIG. 2. Overview of (a) the comparator feedback and (b) DSP feedback used to obtain amplitude stabilization. Phase shifters are labeled with $\phi$, and filter bandwidths (BW) are indicated.
A noiseless feedback drive passing through a comparator generates a fixed oscillation amplitude $A_{o}$. Thus $G(A)=A_{o} / A$ and Eq. (4) together yield

$$
\dot{A}=-\frac{1}{2} \gamma_{z}\left(A-A_{o}\right) \text {. }
$$

The amplitude $A$ damps exponentially to $A_{o}$; the time constant $\gamma_{z} / 2$ is the same as for damping without feedback. Noise injected into a comparator softens its gain response, limiting the gain at low $A$ [9]. Narrow band filters [Fig. 2(a)] to reduce the noise are thus essential. A big challenge is in adjusting the trapping potential to keep the shifting oscillation frequency centered on the filters. [The drives to two electrodes in Fig. 2(a) are effectively one drive insofar as the electron mixes the two frequencies [7].]

We program the DSP chip to calculate a running Fourier transform of the amplified induced signal and to adjust the feedback gain as a cubic function of the largest transform amplitude, so

$$
G(A)=1+a_{1}\left(A-A_{o}\right)+a_{2}\left(A-A_{o}\right)^{2}+a_{3}\left(A-A_{o}\right)^{3} .
$$

For this demonstration only the linear term is used, with $a_{2}=a_{3}=0$. The effective bandwidth (related to the Fourier transform bin width) is $8 \mathrm{~Hz}$, but the "filter" is always centered on the oscillation frequency. No square wave is generated, so no filtering of harmonics is required.

Figure 3 shows that $\omega_{z}\left(A_{o}\right)-\omega_{z}$ depends approximately quadratically upon $A_{o}^{2}$ as predicted by Eq. (3) for $A \ll d . A_{o}$ is varied by changing the gain for three different $V_{c} . A_{o}$ is determined from the size of the induced signal to which it is proportional, with a proportionality constant that will be discussed shortly. Fits of Eq. (3) to the measurements in Fig. 3 allow us to determine and adjust $C_{4}$ and $C_{6}$ with unprecedented accuracy.

One consequence is that extremely small frequency shifts can be quickly detected with the SEO. In a given averaging time, a frequency can typically be measured to the familiar limit provided by the uncertainty principle, divided by the signal-to-noise ratio $(S / N)$ [10]. A large induced $S$ is possible due to the large oscillation amplitudes, illustrated in Fig. 3. The effect of amplitude fluctua-

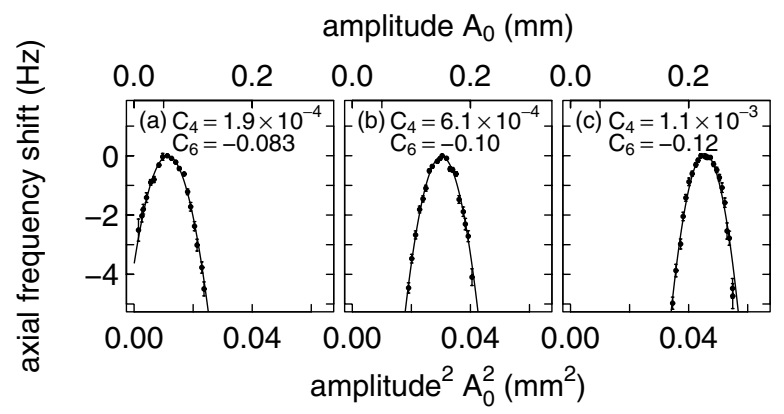

FIG. 3. The axial frequency measured as a function of the square of the axial amplitude $A_{o}$ using the comparator SEO. 
tion noise $N$ (driven by thermal fluctuations in the detection resistor) is particularly small if the oscillator amplitude is stabilized at a maximum (e.g., Fig. 3) caused when the effects of $C_{4}$ and $C_{6}$ of opposite sign cancel. An oscillator is locally harmonic at such maxima, with the oscillation frequency insensitive to small, noise-driven, amplitude fluctuations, despite the large oscillation in an anharmonic potential. Figure 4(a) shows the standard deviation of repeated frequency measurements as a function of averaging time. With only $4 \mathrm{~s}$ of averaging time a five parts in $10^{10}(0.5 \mathrm{ppb})$ shift in $\omega_{z}$ can be measured-a substantial improvement on any other method.

In principle, a fixed frequency drive could sustain a large oscillation in an anharmonic potential. In practice, however, if the oscillator frequency changes suddenly, the oscillation could be lost. Also, a fixed frequency drive cannot generally build up a large oscillation amplitude in the first place, since the oscillator shifts out of resonance with the drive as the oscillation amplitude increases.

The considerable advantage of a SEO is that its selfderived drive always stays resonant, even if its oscillation frequency changes suddenly. It also stays resonant while the oscillation amplitude builds up to a large value, during which time the oscillation frequency is shifting. Typically, our SEO is excited in less than $1 \mathrm{~s}$.

A calibration of the axial oscillation amplitude - using quantum jump spectroscopy of an orthogonal cyclotron motion - is next. Noise applied to the oscillator gives a distribution of axial energies $E_{z}$ about the stable oscillation energy $E_{o}$, amplitude $A_{o}$, and phase $\phi_{o}$ that pertain for no noise. A reservoir at temperature $T_{z}$, weakly coupled to the axial motion, gives a distribution [11]

$$
P\left(E_{z} ; E_{o}, T_{z}\right)=\frac{1}{k T_{z}} e^{-\left(E_{z}+E_{o}\right) /\left(k T_{z}\right)} I_{0}\left(\frac{2 \sqrt{E_{z} E_{o}}}{k T_{z}}\right),
$$

where $I_{0}$ is a modified Bessel function. No feedback drive gives $E_{o} \rightarrow 0$ and a Boltzmann distribution of $E_{z}$.
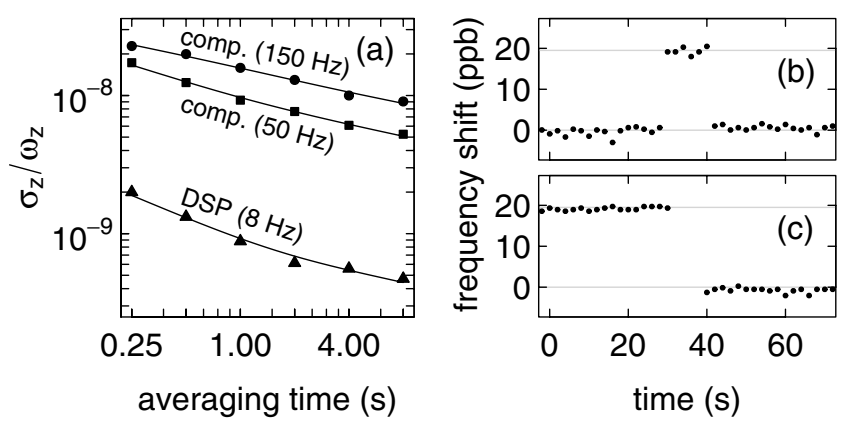

FIG. 4. (a) Fractional standard deviation of repeated frequency measurements for SEOs with indicated bandwidth. Small shifts in the frequency of a $200 \mathrm{MHz}$ SEO indicate (b) a one-quantum cyclotron excitation and (c) a separate spin flip. The SEO is off while a drive is applied to flip the spin, giving the gap in (c).
A simple derivation verifies this distribution and highlights the assumptions. For small fluctuations from $A_{o}$ the oscillation can be taken as harmonic at angular frequency $\omega_{o}=\omega_{z}\left(A_{o}\right)$, with $E_{o}=\frac{1}{2} m \omega_{o}^{2}\left|A_{o} e^{i \phi_{o}}\right|^{2}$. Noise alone would drive the oscillator into thermal equilibrium, to a Boltzmann distribution of energies $E_{n}=\frac{1}{2} m \omega_{o}^{2}\left|A_{n} e^{i \phi_{n}}\right|^{2}$, where $\phi_{n}$ is a random oscillation phase. The oscillation amplitude $A_{z}$ and phase $\phi_{z}$ due to independent feedback and noise drives is the superposition $A_{z} e^{i \phi_{z}}=A_{o} e^{i \phi_{o}}+$ $A_{n} e^{i \phi_{n}}$ for a harmonic oscillation. The combined effect of feedback and noise for a particular $\phi_{n}$ arises from the distribution of the total amplitude

$$
\tilde{P}\left(A_{z} e^{i \phi_{z}}\right) \propto e^{-E_{n} /\left(k T_{z}\right)}=e^{-\left(m \omega_{o}^{2}\left|A_{z} e^{i \phi_{z}}-A_{o} e^{i \phi_{o}}\right|^{2}\right) /\left(2 k T_{z}\right)} .
$$

The probability distribution of $E_{z}=\frac{1}{2} m \omega_{o}^{2}\left|A_{z} e^{i \phi_{z}}\right|^{2}$ in Eq. (7) is the average of this distribution over random $\phi_{z}$.

Remarkably, quantum jump spectroscopy directly measures Eq. (7) and thus determines $A_{o}$ and $T_{z}$. The quantum jumps [12] are between the ground and first excited states of cyclotron motion at frequency $\nu_{c}=148 \mathrm{GHz}$ in a $B=$ 5.24 T magnetic field $B \hat{\mathbf{z}}$. A small "magnetic bottle" gradient $\Delta \mathbf{B} \sim z^{2} \hat{\mathbf{z}}$ [13] from two nickel rings (Fig. 1) weakly couples the cyclotron (or spin) magnetic moment $\mu$ to the axial motion, adding a coupling term that goes as $\mu \Delta B \sim \mu z^{2}$ to the Hamiltonian.

The corresponding small addition to the oscillator's restoring force, $\sim \mu z$, shifts the observed axial oscillation frequency in proportion to $\mu$. Our frequency resolution makes it possible to observe that $\omega_{z}$ shifts by $\delta$ for a single quantum excitation from the cyclotron ground state [Fig. 4(b)]. The probability $P_{c}$ that a cyclotron driving force at a frequency $\nu$ causes a quantum jump [12] thus becomes measurable. For the $200 \mathrm{MHz}$ oscillator, $\delta=$ $3.9 \mathrm{~Hz}$. For the $64 \mathrm{MHz}$ oscillator, $\delta=12 \mathrm{~Hz}$.

The second consequence of the magnetic bottle coupling is that the magnetic field averaged over an axial oscillation changes with oscillation energy, shifting $\nu_{c}$ by $\delta$ for every

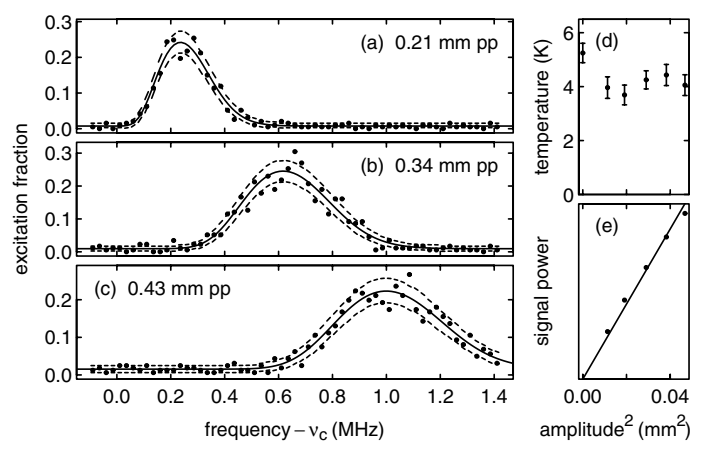

FIG. 5. (a)-(c) Measured quantum jump spectra (points), fits to $P_{c}\left(\nu ; E_{o}, T_{z}\right)$ (solid lines) and $68 \%$ confidence limits (dashed lines) for the comparator SEO conditions of Figs. 3(a)-3(c). (d) $T_{z}$ from the fits. (e) Induced signal (in arbitrary units) is proportional to $A_{o}^{2}$ from fits. 
quantum of axial energy. The quantum jump spectrum,

$$
P_{c}\left(\nu ; E_{o}, T_{z}\right) \propto P\left[\hbar \omega_{z}\left(\nu-\nu_{c}\right) / \delta ; E_{o}, T_{z}\right],
$$

thus reveals the distribution of axial energies of Eq. (7).

Figures 5(a)-5(c) show examples for the three axial oscillation amplitudes that result for the trap settings of Figs. 3(a)-3(c). The extracted temperature $T_{z}$ [Fig. 5(d)] is independent of oscillation amplitude. The detection amplifier makes $T_{z}$ hotter than the ambient temperature, emphasizing that either this amplifier must be off or feedback cooling must be applied [5] to achieve low axial temperatures. Figure 5(e) shows that the extracted $E_{o} \sim A_{o}^{2}$ is proportional to the induced signal power, which can be measured in only seconds. The quantum jump spectroscopy in Fig. 5 which calibrates this signal took about $40 \mathrm{~h}$.

The one-electron SEO allows such good detection of small frequency shifts that a likely application is the measurement of electron and positron magnetic moments - to provide the most accurate direct lepton $C P T$ test, and the most accurate determination of the fine structure constant $\alpha$. Figures 4(b) and 4(c) illustrate the detection of a onequantum cyclotron excitation and a spin flip. Quantum jump spectroscopy-measuring the number of quantum jumps as appropriate drive frequencies are changedcould provide the first fully quantum measurement of these moments.

Averaging the frequency of a one-antiproton $(\bar{p})$ SEO over a long time, to detect extremely small $\delta$, may make possible the long-time goal [14] of measuring the $\bar{p}$ magnetic moment. Improving the $0.3 \%$ current accuracy [15] by a factor of a million or more seems conceivable. The needed $\nu_{c}$ can already be measured to such an accuracy [16]. Measuring the needed spin precession frequency requires observing a $\bar{p}$ spin flip. The $\delta$ that would signal such a flip is proportional to $\mu / \sqrt{m}$ for a particle with magnetic moment $\mu$ and mass $m$. The challenge is that $\mu$ for the $\bar{p}$ is 658 times smaller than that of the electron, and $\sqrt{m}$ is 43 times larger, so that $\delta$ is a daunting $3 \times 10^{4}$ smaller than that of an electron in the same trap.

Fortunately, the size of the frequency shift $\delta$ can be increased, since $\delta$ is proportional to an apparatus factor $\beta M /\left(d \sqrt{V_{o}}\right)$ [7]. For example, making the ring electrode in the trap of Fig. 1 out of iron rather than copper would increase the product of a relative geometry factor $\beta$ and the magnetization $M$ for the magnetic material (and hence $\delta$ ) by a factor of 16. Substantial additional increases could come from reducing the trap size and potential, $d$ and $V_{o}$, limited by the extent to which this makes a more anharmonic axial oscillation. The fractional stability required in the trapping potential goes as $\mu \beta M / V_{o}$ and seems possible. To avoid broadened resonances, spin flips and cyclotron excitations would be made in a trap without a magnetic gradient, then transferred to a detection trap with a large magnetic gradient, as in measurements of magnetic moments of bound electrons [17].
In conclusion, self-excitation is demonstrated with the simplest of microscopic oscillators - a single electron suspended in a Penning trap. Both a comparator and a DSP are used to stabilize large, easily observed oscillations that are much larger than noise-driven fluctuations. Despite the anharmonic trap potential, with the right choice of feedback gain, the SEO rapidly excites itself to a large oscillation that is locally harmonic - with an oscillation frequency largely independent of amplitude fluctuations. It maintains the large oscillation even when its oscillation frequency shifts suddenly. The great signal-to-noise ratio observed with the SEO makes it possible to detect small frequency shifts quickly. The SEO could thus enable better measurements of the electron and positron magnetic moments. It may also make it possible to detect antiproton spin flips for the first time, thereby opening the way to greatly improved measurements of the antiproton magnetic moment.

We are grateful for wonderful electronics support from $\mathrm{J}$. MacArthur and for lab assistance from A. Steinert and O. Elliott. This work was supported by the NSF. B. D. was also supported by the Fannie and John Hertz Foundation, and D. H. by the ARO.

*Current address: Oak Ridge National Laboratory, Oak Ridge, TN 37831, USA.

${ }^{\dagger}$ Current address: California Institute of Technology 12-33, Pasadena, CA 91125, USA.

[1] A. Bain, Great Britain Patent No. 8783 (1841).

[2] T. R. Albrecht, P. Grütter, D. Horne, and D. Rugar, J. Appl. Phys. 69, 668 (1991).

[3] A. N. Cleland and M. L. Roukes, Nature (London) 392 , 160 (1998).

[4] H. Dehmelt, W. Nagourney, and J. Sandberg, Proc. Natl. Acad. Sci. U.S.A. 83, 5761 (1986).

[5] B. D’Urso, B. Odom, and G. Gabrielse, Phys. Rev. Lett. 90, 43001 (2003).

[6] G. Gabrielse and F. C. Mackintosh, Int. J. Mass Spectrom. Ion Process. 57, 1 (1984).

[7] L. S. Brown and G. Gabrielse, Rev. Mod. Phys. 58, 233 (1986).

[8] M. Lax, Phys. Rev. 160, 290 (1967).

[9] W. B. Davenport, Jr., J. Appl. Phys. 24, 720 (1953).

[10] W. F. Walker, T. K. Sarkar, F. I. Tseng, and D. D. Weiner, IEEE Trans. Instrum. Meas. 31, 239 (1982).

[11] L. Brown, Ann. Phys. (N.Y.) 159, 62 (1985).

[12] S. Peil and G. Gabrielse, Phys. Rev. Lett. 83, 1287 (1999).

[13] R. S. Van Dyck, Jr., P. B. Schwinberg, and H. G. Dehmelt, Phys. Rev. Lett. 38, 310 (1977).

[14] W. Quint and G. Gabrielse, Hyperfine Interact. 76, 379 (1993).

[15] S. Eidelman et al., Phys. Lett. B 592, 1 (2004).

[16] G. Gabrielse, A. Khabbaz, D. S. Hall, C. Heimann, H. Kalinowsky, and W. Jhe, Phys. Rev. Lett. 82, 3198 (1999).

[17] H. Häffner, T. Beier, N. Hermanspahn, H.-J. Kluge, W. Quint, S. Stahl, J. Verdú, and G. Werth, Phys. Rev. Lett. 85, 5308 (2000). 